\title{
MUJERES DEL FORMATIVO. SALUD Y ACTIVIDADES EN SAN JOSÉ MOGOTE, OAXACA, MÉXICO
}

\section{FORMATIVE WOMEN. HEALTH AND ACTIVITIES IN SAN JOSE MOGOTE, OAXACA, MEXICO}

\author{
Martha Elena Alfaro Castro ${ }^{1}$ y Susana Gómez Serafín ${ }^{2}$
}

\begin{abstract}
RESUMEN
En los últimos años, el estudio de poblaciones antiguas ha centrado su interés en los diferentes matices y roles que juegan los individuos dentro de una sociedad particular poniendo de relieve las relaciones de género al interior de la estructura social. En este sentido, este trabajo pretende realizar inferencias iniciales de algunos aspectos que pudieron haber formado parte de la cotidianeidad de las mujeres que habitaron la aldea Formativa de San José Mogote. El análisis osteológico de patologías e indicadores de estrés ocupacional llevado a cabo, evidenció algunas diferencias notables por sexo y edad de estos rasgos en los individuos analizados siendo factible que las variaciones encontradas pudieran tener, en parte, relación con una susceptibilidad diferencial con base al sexo, modo de vida o división del trabajo.
\end{abstract}

Palabras clave: San José Mogote, Oaxaca, Salud, Actividad, Mujeres

\section{ABSTRACT}

In recent years, the study of ancient populations has focused its interest in the different nuances and roles played by individuals within a particular society emphasizing gender relations within the social structure. In this sense, this research tries to make initial inferences of some aspects that may have been part of the daily lives of women who lived in the village of San José Formative Mogote. The osteological analysis of pathologies and indicators of occupational stress showed some notable differences by gender and age of these traits in the individuals analyzed is feasible that variations may be found, in part, related to a differential susceptibility based on sex, lifestyle or division of labor.

Keywords: San José Mogote, Oaxaca, Human Health, Occupational Activity, Women

Tipología: Artículo de Investigación Científica y Tecnológica

Fecha de recepción: 20/03/2014

Fecha aceptación: 28/08/2014

Forma de citar este artículo: Alfaro, M. E., \& Gómez, S. (2014). Mujeres del formativo. Salud y actividades en San José Mogote, Oaxaca. Jangwa Pana, 13, 82 - 99

1. Profesor investigador Centro INAH Baja California. Maestra en Antropología física por la Escuela Nacional de Antropología e Historia, México. Correo: ollin_tochtli@yahoo.com.mx

2. Profesor investigador Centro INAH Morelos. Doctorado en Antropología por la Escuela Nacional de Antropología e Historia. México. Correo: xonaxi8@gmail.com 


\section{INTRODUCCIÓN}

\begin{abstract}
$\mathrm{A}_{\mathrm{r}}^{\mathrm{c}}$ ctualmente existen diversas corrientes y posturas en torno a los estudios de género e identidad en arqueología, así como los diferentes mecanismos por medio de los cuales pueden realizarse aproximaciones de género en poblaciones del pasado ${ }^{3}$ En esta presentación no pretendemos ahondar en estos debates ni promover una discusión teórica sobre los mismos; nuestro interés se centra en algunos de los postulados más aceptados de la perspectiva de género y analizar las posibles variaciones en condiciones de salud y estrés ocupacional de los antiguos pobladores de la aldea del Formativo de San José Mogote. ${ }^{4}$ Partimos del análisis biológico de los restos óseos, así como de algunos de los datos arqueológicos, ${ }^{5}$ etnohistóricos y etnográficos de la región para intentar una aproximación a algunos aspectos de la vida cotidiana de las mujeres de esta aldea.
\end{abstract}

En antropología, el sexo suele definirse comúnmente con base en características biológicas consideradas invariables entre hombres y mujeres, determinadas éstas al momento de la concepción y se manifiestan en el subsecuente desarrollo fisiológico. Las diferencias sexuales incluyen rasgos como los cromosomas, los genitales y otras estructuras anatómicas relacionadas con el desarrollo sexual secundario; en casos arqueológicos estas distinciones son asignadas principalmente a través de rasgos físicos en los huesos como el cráneo y la pelvis. Mientras que el género se entiende como una construcción cultural, que cla-

\footnotetext{
3. Un mayor detalle de algunos de principales enfoques teóricos puede revisarse: Wiesheu, 2006; Rodríguez, 2007a; Nelson, 2004; Díaz-Andreu et al, 2005; González, 2007.

4. Estos datos son un avance de los resultados obtenidos de este sitio dentro del proyecto de investigación "estrategias de subsistencia y sus implicaciones biológicas y culturales".

5. Vale la pena señalar que a pesar de que los materiales fueron excavados por J. Marcus y K. Flannery a partir de 1966, a la fecha no sé cuenta con un informe arqueológico que proporcione la información completa de campo, ni los datos concretos del contexto arqueológico de todos los entierros recuperados en el sitio. Por lo que para este trabajo nos basamos en la información arqueológica y las interpretaciones publicadas hasta este momento por los arqueólogos estadounidenses responsables de su exploración.
}

sifica socialmente a los individuos en categorías como masculino o femenino en una cultura dada (Armelagos 1998).

De lo anterior se desprende la importancia que representan ambas categorías ya que la polémica radica en la forma en la que el sexo y el género se vinculan o no con el cuerpo físico. Sin embargo, el cuerpo se percibe a través de la interpretación social por lo que se encuentra estrechamente relacionado con el género, de manera que es difícil entender el género sin el sexo y viceversa. Por otro lado, el cuerpo es mucho más que sexo biológico ya que en su plasticidad diferencial se observa la relación de éste con la vida social y el ambiente, por lo que los esqueletos podrían considerarse, en cierta medida, un producto de la acción humana en la misma manera que lo son otras formas de cultura material y en este sentido actuar como un punto de referencia en el que las interpretaciones del contexto arqueológico pueden realizar algunas inferencias sociales (Soafer, 2006).

La perspectiva de género en bioarqueología más que resaltar el papel de las mujeres en oposición al de los hombres, reconoce la diversidad de géneros y el rol que juegan éstos en la construcción de la sociedad o la forma cómo influyen en otras instituciones culturales, v.gr. patrones de parentesco, actividades económicas, estructura política, creencias religiosas, entre otras (RodríguezShadow, 2007a). Este trabajo no tiene intención de reconstruir o interpretar la población total del período, nos interesa más bien entender en la medida de lo posible qué factores podrían haber estado incidiendo en la salud de las mujeres de San José Mogote durante la etapa aldeana, así como comprender el contexto en el que pueden manifestarse diferencias, esto con base a la consideración de ciertos indicadores asociados a condiciones de salud y estrés ocupacional en restos óseos y con apoyo de las fuentes etnohistóricas. 


\section{San José Mogote (SJM)}

Durante los primeros años del periodo Formativo (alrededor de 1500 a. C), los conjuntos de personas que vivían de forma trashumante, comienzan a establecerse en aldeas permanentes lo que trae aparejada la diversificación lingüística con otros grupos más alejados entre sí. La posibilidad de establecerse a la orilla de los ríos, con los años también propicia el desarrollo de una incipiente agricultura dando ventaja con respecto a otros aldeanos menos favorecidos. La historia del asentamiento de San José Mogote (en adelante $\mathrm{SJM})$ da claras muestras de un rápido crecimiento que la distingue por su mayor tamaño y jerarquía social de entre las siete aldeas del mismo periodo (Fernández y Gómez, 1997) llegando a consolidarse en el centro del poder regional más destacado del Valle de Etla (Figura 1).

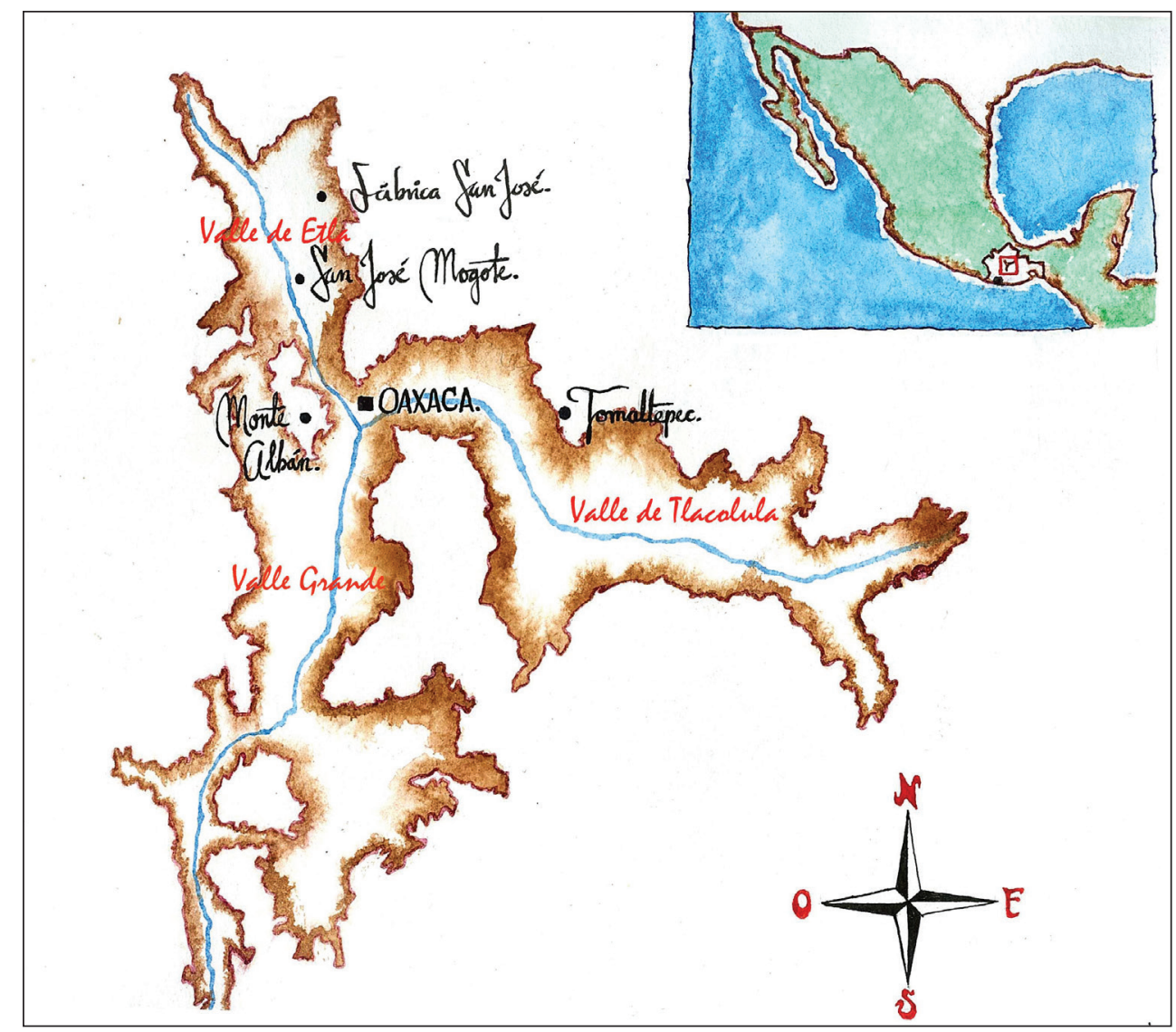

Figura 1. Ubicación de los sitios en los Valles Centrales (Dibujo: Ernesto Arrona Santiago).

Lo anterior vendría acompañado seis siglos después de una incipiente jerarquización social ${ }^{6}$, amparada por la especialización artesanal que permite a ciertos sectores de la población intercambiar y acceder a bienes de consumo por medio de redes de comercio con lejanas regiones como Chalcatzingo, en

6. Flannery y Marcus (2005) localizaron algunas áreas y unidades domésticas que mostraban evidencia de especializaciones artesanales como: la elaboración de adornos de magnetita, concha y objetos de calcedonia. 
el actual estado de Morelos y con San Lorenzo Tenochtitlan, en el actual estado de Veracruz. ${ }^{7}$ Durante 1200-800 a.C., la sociedad estaba constituida por grupos tribales y clanes familiares que al parecer se conformaban por el prestigio, la edad y la experiencia lograda, lo que fue evidente por los tipos diversificados de inhumación que se efectuaban entonces. Para esta época hay certeza de una diferenciación social a la que paulatinamente se incorporan los grupos de hechiceros, sacerdotes y personajes que tienen mayor control político-ideológico e implementan entes sobrenaturales de los que dicen descender.

A través de ritos y cosmogonías que les permite asociarse con esos antepasados mitológicos de los que presume tener su procedencia, la elite gobernante se vincula con los aspectos sobrenaturales de la tierra y el cielo e incorpora tanto en su atuendo como en sus bienes las alegorías del Terremoto y la Tierra (Xoo), del Rayo o lluvia (Niza quiye), del Agua (Cociyo), y de las Nubes $(\mathrm{Zaa})$ entre otras fuerzas cosmogónicas o deidades tutelares que coloca a los gobernantes como mediadores entre su comunidad y lo sobrenatural (Marcus y Flannery, 2001, 111).

\section{Sistema de enterramiento y culto a los ancestros}

En la etapa del Formativo temprano llamada fase Tierras Largas (1400-1150 a.C., la mayoría de los entierros eran depositados en forma extendida dentro de fosas simples en los patios de las unidades domésticas, acompañados algunos con una o más vasijas de barro. Sin embargo, de esta fase se distinguen tres sujetos masculinos que a diferencia del resto, fueron colocados en posición sedente con las extremidades fuertemente flexionadas y acompañados de una ofrenda relativamente más rica. Marcus y Flannery (2001) comentan con base en la posición corporal y su

7. El análisis químico de los espejos de ilmenita de esta aldea ha permitido saber que estos llegaban a comunidades lejanas como Morelos y las Costas del Golfo, y a su vez intercambiaban objetos como espinas de mantarraya, piedras semipreciosas, concha y obsidiana, entre otros bienes (Pires-Ferreira, 1976; Winter, 1990). relación con otros entierros de la región, que dichos sujetos posiblemente pudieron haber sido personas de cierta importancia para la aldea.

Los mismos investigadores indican que para la fase San José (1150-850 a.C.) de mediados del Formativo, se observa un cambio en el sistema de enterramiento en esta aldea y en otros caseríos de los Valles Centrales. ${ }^{8}$ Se Comienza a observarse una mayor frecuencia de entierros múltiples de "marido-mujer" o "familiares", que reemplaza el patrón anterior en el que únicamente los varones eran inhumados con vasijas vinculadas con los ancestros cosmogónicos de Tierra o Cielo. Dando la apariencia que en este momento pudiera ser más importante destacar la pertenencia de una persona a una élite familiar que a una línea masculina específica descendiente de un linaje divino. La distribución de esos espíritus celestiales, al parecer vinculada con los personajes masculinos eran, de acuerdo con Marcus y Flannery (2001), motivos excluyentes de acuerdo con su distribución geográfica; esto significa que cada aldea debió haber sido descendiente de un ancestro celestial, ya fuera del "Cielo" o de la "Tierra".

Los entierros de la fase San José fueron depositados principalmente en posición extendida, con los brazos cruzados. Casi todos los adultos tenían una cuenta de jade en la boca y eran acompañados con una o más vasijas de cerámica, algunas de ellas asociadas sólo a los hombres que formaban parte de la representación del Rayo (fuerzas celestes) y con cantidades variables de jade, magnetita, concha marina o cerámica fina. La diferencia en cuanto a jerarquía social parecía estar más bien indicada por la posición del individuo al momento de su inhumación, ya fuera en posición sedente (postura de autoridad) o en posición extendida con los brazos cruzados sobre el pecho y boca abajo (postura de obediencia) (Marcus y Flannery 2001).

8. Como los asentamientos del Formativo de Tierras Largas, Fábrica San José y Santo Domingo Tomaltepec (Marcus y Flannery, 2001). 


\section{MATERIALES Y MÉTODOS}

El material esquelético humano estudiado procede de exploraciones realizadas desde 1966 en el sitio arqueológico San José Mogote (SJM), por los arqueólogos estadounidenses Kent V. Flannery y Joyce Marcus dentro del proyecto "Prehistoria y Ecología Humana del Valle de Oaxaca". En este trabajo presentamos el análisis de los individuos que han sido fechados para el período Formativo Oaxaqueño ${ }^{9}(1400$ a. C. a 200 a. C.) ya que es la etapa mayormente representada en la colección ósea y los arqueólogos sugieren, que SJM fue ocupado tan solo esporádicamente durante el clásico y posclásico.

La asignación de sexo se realizó a partir de rasgos morfoscópicos basados en los cambios relacionados con la madurez sexual en huesos como cráneo, mandíbula, coxal y sacro; la estimación de la edad biológica de individuos entre recién nacidos y 15 años (subadultos) se efectuó mediante el método de brote dental, el grado de cierre de las epífisis y la longitud de huesos largos. En individuos mayores de 15 años (adultos) la edad biológica se valoró con base en las modificaciones de la superficie auricular del ilíaco y en la sínfisis púbica con la edad (Ubelaker, 1989; Ortega, 1998; White y Folkens, 2005).

Para el análisis se retomó parte de la propuesta teórico-metodológica del proyecto "Historia de la salud y la nutrición en el hemisferio occidental" coordinado por Steckel y Rose (2002), ${ }^{10}$, que considera bajo un enfoque biocultural la evaluación de una serie de indicadores paleopatológicos asociados frecuentemente con condiciones de salud y nutrición en poblaciones del pasado, que evalúan la presencia y frecuencia de alteraciones patológicas así como el grado de afectación.

9. Se integran aquí los entierros de este sitio fechados para las fases del Formativo temprano, medio y tardío.

10. Actualmente se encuentran publicados múltiples trabajos que consideran esta metodología e indicadores y el registro estandarizado de los mismos permite la contrastar los datos obtenidos con otras poblaciones. Los pormenores de esta propuesta teórica-metodología pueden verse en la obra referida arriba.
Se consideraron en esta ocasión solamente los siguientes: criba orbitaria, hiperostosis porótica, hipoplasia del esmalte, patologías bucales como las caries o los abscesos, procesos infecciosos, lesiones degenerativas de tejido articular y traumatismos, los cuales se evaluaron con base en su presencia, frecuencia o severidad (Goodman y Martin, 2002 ${ }^{11}$. Se examinó además un indicador de estrés ocupacional como: el desgaste dental no relacionados con la alimentación. Este rasgo se registró con base a su presencia o ausencia, así como el grado de severidad y el patrón en el que se exhibía (Alfaro, 2006).

La criba orbitaria y la hiperostosis porótica son alteraciones que se observan sobre la superficie del cráneo como una especie de puntilleo resultado de la hiperplasia medular que provoca el adelgazamiento del tejido compacto y el engrosamiento del diploe. Este tipo de lesiones si bien son de etiología multifactorial, en el caso de poblaciones antiguas suelen asociarse con frecuencia a una dieta inadecuada o una absorción pobre de hierro ocasionada por ejemplo por enfermedades gastrointestinales que pueden propiciar la presencia de anemias (Stuart-Macadam, 1989; Blom et al, 2005).

Al ser la hipoplasia una alteración que se presenta en la formación del esmalte, da como resultado interrupciones que se observan a manera de bandas o líneas horizontales, y entre las causas encontramos anomalías congénitas -en estos casos se afecta la corona de forma severa-, traumatismos localizados -que afectan una pequeña porción de la corona de un diente o algunos de los adyacentes-, problemas infecciosos o parasitarios que dificultan o afectan la absorción de nutrientes o estrés nutricional ${ }^{12}$, siendo estas dos últimas las causas más comunes en poblaciones prehispánicas (Marrodán et al, 1995).

11. Debido al estado de conservación de los materiales y la destrucción de los segmentos o piezas clave para la observación de los indicadores hizo variar la "n".

12. Un estado nutricional pobre disminuye la función inmunológica y predispone a la infección, a su vez las enfermedades pueden disminuir recursos nutricionales. 
Por otro lado, las caries son originadas por la acción de bacterias habituales en la boca como el lactobacillus acidophilus o el streptococcus mutans que al reaccionar con ciertos alimentos favorece la formación de una placa bacteriana que provoca la desmineralización de los dientes y la generación de huecos en los mismos. La formación de este tipo de lesiones se ve influenciado por la interacción de diversas variables como la propia resistencia del individuo, la higiene, el tipo de agente patógeno o qué es lo que se come (p.e. la textura o composición química de los alimentos), por lo que su presencia y severidad en poblaciones pretéritas puede darnos pistas también sobre la dieta o hábitos de higiene. La extensión de caries puede producir infecciones en el tejido pulpar, promoviendo la necrosis o formación de abscesos, que de no ser tratados pueden llegar a provocar la pérdida de las piezas dentales (Hillson, 2002; Marrodán et al, 1995).

Mientras que las reacciones periostiales son lesiones infecciosas que ocurren en la superficie de los huesos resultado de la inflamación del periostio, en el caso de las poblaciones antiguas la mayoría de estas reacciones son de naturaleza no especifica (Ortner y Putschar, 1981). En los estudios de salud se emplean para hacer inferencias acerca del impacto en los grupos de riesgo de una población así como los factores ecológicos y culturales que predisponen estas condiciones. Para este indicador se evalúa el grado de involucramiento en tibia, por su posición más superficial y su estructura vascular más sensible a las infecciones, pero se registra también la presencia de lesiones en todo el esqueleto para evidenciar una secuencia sistémica o postraumática.

Las enfermedades degenerativas son una condición patológica progresiva, crónica y no inflamatoria. Se caracterizan por la pérdida de cartílago articular y la subsecuente lesión resultado del contacto inter-óseo en las articulaciones de movimiento libre; las causas pueden ser mecánicas, infecciosas, metabólicas, y edad avanzada, entre otras (Aufderheide y Rodríguez, 1998). Se ha observado una relación entre el es- trés ocupacional y la osteoartris en la realización de ciertas actividades, debido a lo repetitivo de los movimientos y la intensidad de los mismos con el tiempo puede llegar a influenciar un desgaste diferencial en ciertas articulaciones específicas como: columna, codos, manos, en las cuales, llegando a presentarse diferentes grado de afectación en un mismo grupo de edad o sexo (Soafer, 2006; Lai y Lovell, 1992).

Los traumatismos son lesiones o disrupciones del funcionamiento normal del tejido vivo causado por una fuerza externa al cuerpo. Los traumatismos más comunes en hueso, son las lesiones ocasionados por el estrés repentino o gradual (Lovell, 1997). Tanto accidentes como ciertas actividades culturales como las guerras o especializaciones artesanales, pueden favorecer la aparición de lesiones de este tipo, por lo que su estudio proporciona importante información acerca de violencia interpersonal y otros aspectos de la vida cotidiana de poblaciones del pasado. Teniendo en cuenta que en los combates cuerpo a cuerpo suelen presentarse predominantemente traumatismos en la cráneo y región facial -especialmente en huesos nasales y zigomáticos-, así como en costillas y dedos de las manos, mientras que en actividades cotidianas es más frecuente encontrar lesiones en huesos de extremidades superiores e inferiores a consecuencia de accidentes cotidianos o fracturas por fatiga (Herskovitz, et al, 1996; Standen y Arriaza, 2000).

Una vez que los dientes brotan en su posición funcional, las coronas comienzan a ser abrasadas por la atrición con otros dientes, por la comida, las técnicas de preparación de alimentos y otros objetos que son colocados en la cavidad oral usando los dientes para sujetarlo. Por lo que en este trabajo se realizó un análisis de las modificaciones observadas en los dientes que pudieran no estar relacionados con el patrón de masticación o con alguna práctica cultural como la mutilación dentaria; la utilización de los dientes como apoyo para la realización de algunas actividades como el ablandar las pieles para la fabricación de ob- 
jetos o el alisar fibras vegetales para la elaboración de cestería, suelen dejar una serie de patrones específicos, principalmente en la zona oclusal o interproximal, dando como resultado facetas aplanadas y ranuras o surcos en las áreas de contacto (Smith, 1972; Lukacs y Pastor, 1988).

\section{RESULTADOS Y DISCUSIÓN}

Se examinaron 42 individuos de esta colección ósea ${ }^{13}$ de distintas edades y de uno y otro sexo, con un estado de conservación que varía de malo a bueno (Figura 2).

La distribución por sexo y edad de los individuos analizados es la siguiente: $40.5 \%$ (17/42) son mujeres, 28.6\% (12/42) hombres, 9.5\% (4/42) adultos que por el mal estado de conservación de elementos diagnósticos para la asignación de sexo no fue posible distinguirlo y 21.4\% (9/42) subadultos (Tabla 1).

Tabla 1. Distribución de los individuos por sexo y grupo de edad.

\begin{tabular}{|c|c|c|c|c|c|}
\hline \multirow{2}{*}{ Período } & \multicolumn{3}{|c|}{ Adultos por sexo } & \multirow[b]{2}{*}{ Subadultos*** } & \multirow[b]{2}{*}{ Total } \\
\hline & Femeninos & Masculinos & Adultos** & & \\
\hline Formativo & $\begin{array}{c}17 / 42(40.4 \%) \\
\text { Grupo de edad: } \\
18-20(4) \\
21-35(7) \\
36-55(5) \\
\text { Adulto* (1) }\end{array}$ & $\begin{array}{c}\text { 12/42 (28.5\%) } \\
\text { Grupo de edad: } \\
18-20(2) \\
21-35(3) \\
36-55(5) \\
\text { Adulto* (2) }^{*}\end{array}$ & $\begin{array}{c}\text { 4/42 (9.5\%) } \\
\text { Grupo de edad: } \\
21-35(2) \\
\text { Adulto* (2) }\end{array}$ & $\begin{array}{c}9 / 42(21.4 \%) \\
\text { Grupo de edad: } \\
0-3(3) \\
4-7(1) \\
12-17(5)\end{array}$ & $\begin{array}{l}42 / 42 \\
100 \%\end{array}$ \\
\hline Total & 17 & 12 & 4 & 9 & $42(100 \%)$ \\
\hline
\end{tabular}

* Individuos adultos que pudo determinarse el sexo pero no la edad.

** Individuos adultos que no fue posible determinar el sexo.

*** Individuos menores de 15 años cuya asignación de sexo no es confiable.

Fuente. Datos propios

\section{Criba Orbitaria, hiperostosis porótica e hipoplasia del esmalte}

En los individuos analizados del período Formativo de esta aldea, notamos que tanto la criba $(25.0 \% 6 / 24)$ como la hiperostosis $(28.1 \% 9 / 32)$ se presentan predominantemente en su forma leve en porcentajes relativamente bajos con independencia de la edad, pero con una ligera superioridad en mujeres con respecto de los hombres (Tabla 2). En el caso de la criba orbitaria, ésta se observó en $18.1 \%(2 / 11)$ de mujeres, $12.5 \%$ (1/8) de hombres y en el

13. Esta colección se encuentra bajo resguardo del Centro INAH Oaxaca desde los años ochenta. 
caso de los subadultos en $20 \%$ (1/5) de los sujetos que presentaban la región anatómica para la evaluación de este indicador se vieron afectados. Por otro lado la hiperostosis porótica se localizó en un $33.3 \%(5 / 15)$ de las mujeres, $30 \%(3 / 10)$ de los hombres, $20 \%(1 / 5)$ de los subadultos y $0 \%(0 / 2)$ de los adultos que debido al estado de conservación no fue posible identificarse el sexo (Tabla 2).

La hipoplasia se presentó de igual manera con una baja incidencia en los individuos analizados, independientemente de la edad los mismos $(28.1 \% 9 / 31)$ pero con una leve diferencia respecto al sexo, encontrándose esta lesión en sujetos femeninos en un $36.3 \%(4 / 11)$, en masculinos $30 \%(3 / 10)$, en subadultos $28.5(2 / 7)$ y $33.3 \%$ (1/3) en adultos de sexo desconocido (Tabla 2).

Tabla 2. Distribución de indicadores de Salud y Nutrición SJM.

\begin{tabular}{|l|c|c|c|c|c|c|c|c|c|c|}
\hline \multirow{2}{*}{ Indicadores } & \multicolumn{2}{|c|}{ Femeninos } & \multicolumn{2}{|c|}{ Masculinos } & \multicolumn{2}{|c|}{ Subadultos } & \multicolumn{2}{|c|}{ Adultos } & \multicolumn{2}{|c|}{ Total } \\
\cline { 2 - 11 } & $\mathbf{n}^{*}$ & $\%^{1}$ & $\mathbf{n}^{*}$ & $\%^{1}$ & $\mathbf{n}^{*}$ & $\%^{1}$ & $\mathbf{n}^{*}$ & $\%^{1}$ & $\mathbf{N}^{* *}$ & $\%^{2}$ \\
\hline $\begin{array}{l}\text { Criba } \\
\text { Orbitaria }\end{array}$ & $2 / 11$ & 18.1 & $1 / 8$ & 12.5 & $1 / 5$ & 20.0 & $0 / 0$ & 0 & $4 / 24$ & 16.6 \\
\hline $\begin{array}{l}\text { Hiper. } \\
\text { porótica }\end{array}$ & $5 / 15$ & 33.3 & $3 / 10$ & 30.0 & $1 / 5$ & 20.0 & $0 / 2$ & 50.0 & $9 / 32$ & 28.1 \\
\hline Hipoplasia & $4 / 11$ & 36.3 & $3 / 10$ & 30 & $2 / 7$ & 28.5 & $1 / 3$ & 3.33 & $9 / 31$ & 29.0 \\
\hline $\begin{array}{l}\text { Periostitis en } \\
\text { tibia }\end{array}$ & $4 / 12$ & 33.3 & $2 / 7$ & 28.5 & $1 / 5$ & 20.0 & $0 / 2$ & 0 & $7 / 26$ & 26.9 \\
\hline $\begin{array}{l}\text { Periostitis en } \\
\text { esqueleto }\end{array}$ & $3 / 15$ & 20.0 & $2 / 12$ & 16.6 & $1 / 7$ & 14.2 & $0 / 3$ & 0 & $6 / 37$ & 16.2 \\
\hline Caries & $10 / 13$ & 76.9 & $8 / 11$ & 72.7 & $0 / 8$ & 0 & $3 / 3$ & 100 & $21 / 35$ & 60.0 \\
\hline Abscesos & $6 / 14$ & 42.8 & $5 / 11$ & 45.4 & $0 / 6$ & 0 & $0 / 3$ & 0 & $11 / 34$ & 32.3 \\
\hline
\end{tabular}

* n representa el número total de individuos del mismo sexo que contaban con el segmento anatómico para el registro del indicador.

** N representa el número total de individuos que contaban con el segmento anatómico para el registro del indicador.

${ }^{1}$ Porcentajes respecto al total de individuos del mismo sexo que presentan la parte ósea para su observación

2 Porcentajes respecto al total de individuos por categoría de indicador.

Fuente: Datos propios

La reducida presencia de patologías como la criba orbitaria (16.6\%), la hiperostosis $(28.1 \%)$ o la hipoplasia del esmalte (29\%) en los sujetos estudiados. Según lo reportado por Flannery Marcus (2005) los datos arqueológicos recuperados en las unidades domésticas de este sitio aportan evidencia de actividades de subsistencia que combinaban de manera intensiva la caza-recolección y agricultura, así como un crecimiento político notable que lo llevo a destacar como un centro político-económico de primer orden para este 
período. Es probable que su distinción política en la región, así como la estrategia combinada de caza-recolección con agricultura, y la excelente ubicación del sitio ${ }^{14}$ pudiera brindar a los pobladores de SJM condiciones propicias para ir cambiando su modo de subsistencia permitiéndoles adaptarse gradualmente a la vida aldeana, y mantener al alcance una gran variedad de alimentos con los que complementar su dieta ${ }^{15}$. En cuanto a las sutiles variaciones encontradas con relación al sexo de los individuos, si bien en culturas del tipo como la aquí estudiada, suele haber una serie de restricciones o acceso diferencial a recursos por cuestiones sociales y de género, no puede dejarse de lado el hecho de que existe también una susceptibilidad biológica diferencial relacionada con el sexo biológico de los individuos ${ }^{16}$ que puede estar de igual manera jugando un papel importante en conjunto con los procesos infeccio$\operatorname{sos}^{17}$ en la asimilación de nutrientes.

\section{Procesos infecciosos}

La incidencia de periostitis en tibia en los sujetos estudiados de esta aldea es relativamente escasa, observándose estas lesiones respectivamente en tan solo un $26.9 \%$ (7/26) y 16.2 (5/37) de los individuos en su forma ligera a moderada ${ }^{18}$, viéndose principalmente afectados por este tipo de padecimientos los individuos adultos jóvenes (21-35 años). Las diferencias por sexo en la frecuencia de estos padecimientos difiere levemente, encontrándose un porcentaje ligeramente mayor en mujeres de periostitis en tibia $33.3 \%(4 / 12)$ y en otros huesos del esqueleto $20 \%(3 / 15)$ con respecto al $28.5 \%(2 / 7)$ y $16.6 \%(2 / 12)$ observado

14. Rodeado de una gran cantidad de tierras de rico potencial agrícola y agua permanente para el cultivo y el uso doméstico (Marcus y Flannery, 2001).

15. La evidencia arqueológica recuperada en unidades domésticas de este período muestra que los antiguos pobladores consumían una gran gama de plantas, frutas y semillas, cuya combinación con carne de algunos animales pequeños..

16. Debido a procesos metabólicos como los ocurridos durante la menstruación, el embarazo y la lactancia, las mujeres llegan también a ser más susceptibles a padecer episodios de anemia (Stuart-Macadam , 1998). 17. Cuando existen problemas nutricionales, como en el caso de la anemia por deficiencia de hierro, se compromete el sistema inmunológico disminuyendo la resistencia a infecciones.

18. Sin llegar a afectar más de la mitad del hueso. en individuos masculinos (Tabla 2). El bajo porcentaje de afectación en los sujetos analizados y el hecho de que se manifieste generalmente en forma leve y cicatrizada, nos hace pensar que a pesar del rápido crecimiento al que se vio sometida la aldea durante el Formativo, ésta pudo haber logrado un cierto equilibrio mediante un adecuado control sanitario, donde la higiene personal, el tipo de vivienda, el control de los deshechos por medio de basureros, entre otros factores, pudieron haber jugado un papel importante en la salud de sus pobladores. En cuanto a las ligeras discrepancias encontradas en la incidencia de infecciones en adultos, estas pudieron deberse a diversos factores, entre ellos la variedad de actividades ocupacionales de la comunidad, que podría quizás exponer de forma dispar a factores infecciosos, así como la susceptibilidad diferencial relacionada con el sexo y la edad que otorgan capacidades distintas a los individuos para resistir las embestidas contra la salud, ya que por ejemplo, durante el embarazo y la lactancia el sistema inmunológico de las mujeres puede verse notablemente afectando incrementando la vulnerabilidad a las infecciones (Armelagos, 1998).

\section{Patologías bucales}

En el caso de los padecimientos bucales como las caries, éstas se manifestaron en un alto número en molares permanentes de los individuos examinados que contaban con piezas dentales, $60 \%$ (21/35); este tipo de patología se encontró únicamente en los individuos adultos mayores de 21 años, $76.9 \%(10 / 13)$ en mujeres, $72.7 \%(8 / 11)$ en hombres y $100 \%(3 / 3)$ en individuos que no fue posible la asignación de sexo (Tabla 2).

Sin embargo, se presentaron diferencias notables en el número de dientes afectados con respecto del sexo, el 46.2\% (6/13) de los sujetos femeninos oscilaban entre 5-9 dientes con caries, mientras que el 54.5\% (6/11) de los hombres tenían entre 1-4 (Figura 2). Por otro lado, los abscesos tuvieron una frecuencia considerable en los individuos, $32.3 \%$ (11/34); esta patología se encontró 
únicamente en sujetos adultos de ambos sexos mayores de 30 años, $42.8 \%$ (6/14) en femeninos y $45.4 \%(5 / 11)$ en masculinos (Figura 4) y puede estar relacionado con el alto contenido de carbohidratos de la dieta, así como con una higiene bucal poco adecuada. Para los dolores de dientes, los cronistas mencionan que los antiguos indígenas oaxaqueños empleaban las hojas de una planta llamada piciete $^{19}$, las cuales secaban al sol y las molían con un poco de cal, esta mezcla la untaban entre las encías y los dientes y la traían durante todo el día, otra hierba que usaban para el dolor de muelas era el olotlpatli ${ }^{20}$ (Acuña, 1984; Del Paso y Troncoso, 1905).

Figura 2. Distribución de No. de dientes con caries por sexo. Fuente: Datos propios

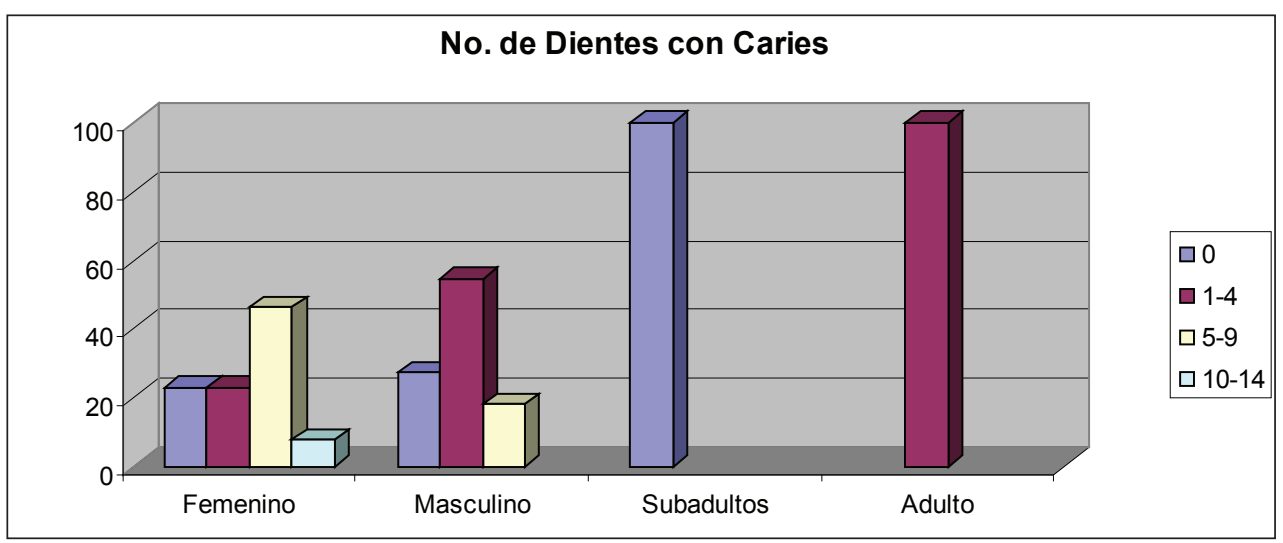

En diversas poblaciones del mundo se ha encontrado un notable incremento de caries asociado al cambio de la dieta de cazadores-recolectores a agricultores $^{21}$, quienes tienen como base de su alimentación granos como el maíz, cuyo almacenamiento permite además su consumo durante la mayor parte del año ${ }^{22}$, en comparación con poblaciones que basan su sustento principalmente en productos de origen marino o las que tienen economías mixtas que combinan pastoreo, pesca y agricultura, en donde su frecuencia es menor (Hillson, 2002).

En el caso de los antiguos mogoteños aquí examinados, el considerable número de sujetos con caries y la cantidad de dientes afectados con esta lesión podría estar relacionado además de un alto consumo de maíz en la dieta, con las técnicas de procesamiento de los alimentos. En la recopilación de cróni-

19. Pedro Franco [1580] menciona que la planta del tabaco o piciete (del náhuatl picietl) era conocido en España como beleño (Franco, en Acuña, 1984). El jugo que se extrae de las hojas del tabaco se mezcla con la cal para facilitar la absorción de los alcaloides de la planta y evitar el cansancio físico y proporcionar bienestar general. Actualmente en varias zonas de Oaxaca los indígenas siguen empleando las hojas de esta planta para diversas curaciones.

20. Del náhuatl olotl, olote (corazón del maíz) y patli, medicina.

21. De una dieta que cuenta con una cierta variedad de carne y menor cantidad de plantas con alto contenido de carbohidratos a una dieta más dura y basada principalmente en cereales (Hillson, 2002).

22. Las caries en esta superficie suelen tener relación además del alto contenido de carbohidratos de los alimentos, con la forma en que estos se consumen, a manera de masas chiclosas que son difíciles de limpiar y favorecen el ambiente para las caries, siendo la zona de la raíz y la interproximal las más afectadas (Idem.) 
cas del siglo XVI que realizan Acuña (1984) y Del Paso y Troncoso (1905) se encuentran múltiples referencias sobre la importancia del maíz en la dieta de los oaxaqueños prehispánicos, así como algunos de los diversos preparados que hacían con él y con otras plantas y semillas, entre las que pueden encontrarse por ejemplo la masa de maíz, cacao y hueso de mamey molido que disolvían en agua y que solían tomar con frecuencia $^{23}$, o el "mixcal" que era una especie de conserva muy dulce de hoja de maguey que se cocía bajo tierra y se comía a tajadas, así como el gusto que tenía por la miel de abeja.

En cuanto a las diferencias en la incidencia de caries, se ha encontrado comúnmente en sociedades agrícolas con dietas basadas en maíz una elevada prevalencia de este padecimiento en mujeres (Armelagos 1998), para lo cual existen una serie de hipótesis que buscan explicar este fenómeno, por un lado, esta el hecho de que generalmente las mujeres suelen estar en relación más estrecha con la recolección de plantas y semillas así como con el procesamiento de los alimentos lo que podría favorecer en ellas una dieta con un contenido mayor de carbohidratos, por otro lado existen también en algunas culturas restricciones en cuanto a la calidad y cantidad de alimentos con base al sexo o edad de los individuos especialmente en épocas de carestía (Speth, 1990; Spiemann, 1989), por último están las diferencias biológicas, las caries pueden llegar a ser más comunes en mujeres jóvenes que en hombres debido a que la erupción dental es más temprana en las primeras lo que permite un mayor tiempo de exposición (Hillson, 2002).

\section{Lesiones degenerativas de tejido articular}

La incidencia de lesiones degenerativas de tipo osteoartrítico en los individuos estudiados oscilan alrededor del 30-40\%, observándose princi-

23. Puede tratarse de una bebida que aún realizan en la actualidad que se conoce como "tejate". Este es un preparado de maíz, hueso de mamey y flor de cacao molido en metate, cuya masa es bañada en agua que suele tomarse en pequeñas jícaras. palmente porosidad y osteofitos iniciales a moderados, siendo las zonas más afectadas: columna, codo, mano y pie (Tabla 3). Sin embargo, se encontraron algunas diferencias interesantes en las regiones involucradas entre los sexos. En el caso de las mujeres se notaron porcentajes más elevados en regiones como: Codo (41.6\% 5/12), rodilla $(40 \% 4 / 10)$, pie $(45.4 \% 5 / 11)$ y la zona torácica $(42.8 \% 3 / 7)$ y lumbar $(50 \% 4 / 8)$. Mientras que en los hombres fueron: hombro (37.5\%), codo $(28.5 \% 2 / 7)$, mano $(42.8 \% 3 / 7)$, cervicales $(37.5 \% 3 / 7)$ y lumbares $(28.5 \% 2 / 7)$.

El patrón de lesiones degenerativas encontrado en las mujeres analizadas podría estar asociado con su participación en algunas actividades como la molienda en metate, la cual era un labor común realizada desde edades tempranas en las poblaciones mesoamericanas para la preparación de alimentos cotidianos y festividades (RodríguezShadow, 1988; 2007b), y en el caso de las mogoteñas no debió haber sido la excepción tomando en consideración la gran cantidad de piedras de molienda y manos de metate que fueron encontradas en el sitio (Flannery y Marcus, 2005), otra de las actividades en la que pudieron haber estado colaborando es la elaboración de cerámica, cestería y tejido ${ }^{24}$, que fueron algunas de las tareas más comunes en el registro arqueológico de las unidades domésticas del sitio y que de igual manera se reportan como asociadas al trabajo femenino en las fuentes etnohistóricas de la región Oaxaqueña (Flannery y Marcus, 2005; Acuña, 1984; Burgoa cfr. en Dahlgren, 1990).

La postura arrodillada en la que se realiza la molienda (de granos y semillas) en metate o la que se toma por ejemplo para amasar la pasta de la cerámica o la que se adquiere mientras se elaboran tejidos en telar de cintura, promueve que algunas articulaciones sufran gran tensión como

24. El tejido es una de las actividades que más que frecuentemente se encuentra asociadas a mujeres en el registro etnohistórico, podemos verla tanto en fuentes iconográficas como en los registros de diversos códices así como en descripciones de algunos cronistas. Herrera (cfr. en Dahlgren, 1990) por ejemplo, refiere que desde el momento del nacimiento a las niñas se les colocaba un huso en la mano. 
la de rodilla o la de metatarso-falanges, debido a que durante estas actividades se requiere que se coloquen en esta postura durante largos períodos de tiempo; así mismo los movimientos repetitivos que se realizan en estas labores pueden estresar considerablemente la articulación de codo o la zona torácica y lumbar. Otra tarea que pudo quizás haber influido en el patrón de lesiones observado en la columna de las mujeres estudiadas pudo haber sido la acción de cargar objetos diversos en la espalda con apoyo de un mecapal colocado a la altura de los hombros -auxiliado con tenates o canasto de palma tejida- para el traslado de diversos artículos como leña, agua o vegetales desde el campo de cultivo a las unidades domésticas, ya que la posición que se adopta para cargar objetos por este medio modifica la curvatura normal de la columna de manera que el peso es transferido a la espalda baja (Dahlgren, 1990; Molleson, 1994; Soafer, 2006).

Tabla 3. Distribución de lesiones degenerativas SJM.

\begin{tabular}{|l|c|c|c|c|c|c|c|}
\hline \multirow{2}{*}{\multicolumn{2}{|c|}{ Región anatómica }} & \multicolumn{2}{|c|}{ Mujeres } & \multicolumn{2}{c|}{ Hombres } & \multicolumn{2}{c|}{ Total } \\
\cline { 3 - 8 } & $\mathbf{N}^{*}$ & $\mathbf{\%}^{1}$ & $\mathbf{n}^{*}$ & $\%^{1}$ & $\mathbf{N}^{* *}$ & $\%^{2}$ \\
\hline \multirow{3}{*}{ Columna } & cervicales & $2 / 8$ & 25.0 & $3 / 8$ & 37.5 & $5 / 16$ & 31.2 \\
\cline { 2 - 8 } & Torácicas & $3 / 7$ & 42.8 & $1 / 7$ & 14.2 & $4 / 14$ & 28.5 \\
\cline { 2 - 8 } & Lumbares & $4 / 8$ & 50.0 & $2 / 7$ & 28.5 & $6 / 15$ & 40.0 \\
\hline Hombro & $1 / 10$ & 10.0 & $3 / 8$ & 37.5 & $4 / 18$ & 22.2 \\
\hline Codo & $5 / 12$ & 41.6 & $2 / 7$ & 28.5 & $7 / 19$ & 36.8 \\
\hline Muñeca & $1 / 9$ & 11.1 & $1 / 5$ & 20.0 & $2 / 14$ & 14.2 \\
\hline Mano & $2 / 10$ & 20.0 & $3 / 7$ & 42.8 & $5 / 17$ & 29.4 \\
\hline Rodilla & $4 / 10$ & 40.0 & $2 / 8$ & 25.0 & $2 / 18$ & 11.1 \\
\hline Pie & $5 / 11$ & 45.4 & $2 / 8$ & 25.0 & $7 / 19$ & 36.8 \\
\hline
\end{tabular}

* n representa el número total de individuos del mismo sexo que contaban con el segmento anatómico para el registro del indicador

** N representa el número total de individuos que contaban con el segmento anatómico para el registro del indicador

${ }^{1}$ Porcentajes respecto al total de individuos del mismo sexo que presentan la parte ósea para su observación

${ }^{2}$ Porcentajes respecto al total de individuos por categoría de indicador

Fuente: Datos propios

Los hombres además del trabajo agrícola y la cacería ${ }^{25}$, pueden haber estado participando en actividades como la elaboración de adornos de magnetita o concha, así como la producción de algunos objetos grandes de piedra o instrumentos de materiales que requerían de mayor fuerza física para su manipulación, en varias de las unidades domésticas de la aldea se encontraron diversos talleres y áreas de actividad de este tipo de implementos (Flannery y Marcus, 2005); labores de este tipo pueden haber favorecido en ellos la presencia de lesiones degenerativas en articulaciones como hombro, codo

25. La cacería en este período parece haberse realizado más bien con redes y lanzas de madera con puntas endurecidas en el fuego (Flannery y Marcus, 2005). 
y mano. Por otro lado, los sujetos masculinos muestran también evidencia de mayor estrés en regiones de la columna como la cervical y la lumbar, lo cual podría estar vinculado con la acción de cargar objetos pesados directamente sobre la cabeza o por medio de un mecapal colocado sobre la frente, durante este período se realizan en el centro de la comunidad múltiples construcciones y los hombres podrían haber estado participando en cierta medida en el acarreo de materiales para construcción; si bien los hombres de esta aldea deben haber colaborado en el sistema de tequio ${ }^{26}$ para la construcción de edificios de la villa, la mayoría de la mano de obra para las edificaciones que se hicieron durante este período pudo haber sido traída de las aldeas localizadas en las cercanías. Es posible también, que los hombres se apoyaran en la extracción de baro y desgrasante y su traslado a la aldea para la elaboración de cerámica ${ }^{27}$, ya que la alfarería es una de las actividades más comunes encontradas en el registro arqueológico, en la cual debieron haber participado todos los miembros del grupo doméstico dentro de algunas de las distintas fases del proceso productivo ${ }^{28}$.

\section{Traumatismos}

La frecuencia de traumatismos en los individuos analizados fue considerablemente muy baja, tan solo se encontraron en mujeres en zonas como el tórax $(4.3 \% 1 / 23)$ y extremidad superior $(8.6 \%$ 2/23) (Tabla 4), los cuales podrían haber sido resultado como se explica más adelante, de accidentes como caídas. En extremidad superior, se encontró una pequeña fractura sanada en la cara posterior de la epífisis proximal del cubito, en el sitio de inserción del tríceps braquial; las

26. Trabajo de tipo colectivo en la comunidad.

27. En el proceso productivo de la alfarería las mujeres de distintas edades participan en la mayor parte de las actividades, mientras que en la extracción participan predominantemente hombres, esposos e hijos de las alfareras, aunque la mujeres solas o viudas pueden en algunos casos integrarse a esta labor (De la Vega et al, 2005).

28. Los niños aprenden inicialmente por imitación el trabajo las madres. En la alfarería las mujeres se inician desde temprana edad, haciendo pequeñas vasijas y juguetes para practicar las técnicas del modelado, pintado y bruñido, una vez que adquieren practica comienzan a hacer ollas y posteriormente las demás formas (De la Vega et al, 2005). fracturas en esta zona son habitualmente resultado de un trauma directo con el codo en flexión o de forma indirecta por una tracción violenta del tríceps, en este caso se observa apenas un ligero desprendimiento de hueso (probablemente de un pequeño espolón del olécranon) por un estirón del tendón del tríceps en un movimiento forzado, es posible que no haya existido alteraciones en la estabilidad de la articulación aunque si debió haber limitado los movimientos de flexión-extensión del antebrazo (Miralles, 2001; Mirralles y Mirralles, 2007).

La otra lesión que se registró en extremidad superior fue en epífisis distal de radio, la cual generalmente es resultado de una caída en la que se apoya todo el peso del cuerpo sobre la muñeca que no es capaz de mantenerse firme y sostener toda la masa corporal (Whiting y Zernicke, 1998); en este caso se observó además la generación de osteofitos ligeros en la cara posterior y la base de este hueso, debido al esfuerzo que debió hacer la articulación al continuar el antebrazo en movimiento, ya que se observaron inserciones moderadas a marcadas de los músculos que participan en los movimientos de prono-supinación y extensión de la muñeca. Mientras que en el caso de la región de tórax, el traumatismo se registró en costillas, encontrándose fracturas sanadas en la epífisis proximal de por lo menos dos costillas (6ta y 7ma) de una mujer de entre 30-34 años. Las lesiones de este tipo generalmente son resultado de un trauma directo, como un golpe o una caída contra un objeto duro (Lovell, 1997).

\section{Desgate dental}

De los 26 individuos que contaban con dentición, únicamente se registraron 3 casos $(11.5 \%)$ de desgastes relacionados con la utilización de los dientes como herramientas: uno femenino y dos masculinos (Tabla 5). Las edades de éstos oscilaban entre 38-50 años, quienes tomando en consideración la esperanza de vida de aldeas de este período se trataría de ancianos; se encontraron en éstos dos tipos de desgaste en mandíbula: 
uno que va en dirección mesial de caninos a incisivos centrales y otro que va en dirección distal del segundo premolar al primer molar, que podrían ser resultado del empleo de la dentición para jalar, sostener o ejercer presión con algún objeto. En poblaciones como los esquimales se han observado desgastes parecidos a los encontrados aquí, ocasionados por el apoyo de la dentición en actividades del procesamiento de pieles, en donde se suelen masticar y estirar las pieles secas de animales para suavizarlas (Merbs, 1983).

Tabla 4. Traumatismo SJM.

\begin{tabular}{|l|c|c|c|c|c|c|c|c|c|c|}
\hline \multirow{2}{*}{ Indicador } & \multicolumn{2}{|c|}{ Mujeres } & \multicolumn{2}{|c|}{ Hombres } & \multicolumn{2}{c|}{ Subadulto } & \multicolumn{2}{|c|}{ Adulto } & \multicolumn{2}{|c|}{ Total } \\
\cline { 2 - 11 } & $\mathbf{N}^{*}$ & $\%^{1}$ & $\mathbf{N}^{*}$ & $\%^{1}$ & $\mathbf{N}^{*}$ & $\%^{1}$ & $\mathbf{N}^{*}$ & $\%^{1}$ & $\mathbf{N}^{* *}$ & $\%^{2}$ \\
\hline Cabeza & $0 / 15$ & 0 & $0 / 10$ & 0 & $0 / 4$ & 0 & $0 / 2$ & 0 & $0 / 31$ & 0 \\
\hline Tórax & $1 / 12$ & 8.3 & $0 / 8$ & 0 & $0 / 3$ & 0 & $0 / 0$ & 0 & $1 / 23$ & 4.3 \\
\hline $\begin{array}{l}\text { Extrem } \\
\text { super. }\end{array}$ & $2 / 12$ & 16.6 & $0 / 7$ & 0 & $0 / 2$ & 0 & $0 / 2$ & 0 & $2 / 23$ & 8.6 \\
\hline $\begin{array}{l}\text { Extrem. } \\
\text { Infer. }\end{array}$ & $0 / 12$ & 0 & $0 / 9$ & 0 & $0 / 2$ & 0 & $0 / 2$ & 0 & $0 / 25$ & 0 \\
\hline
\end{tabular}

* n representa el número total de individuos del mismo sexo que contaban con el segmento anatómico para el registro del indicador

** $\mathrm{N}$ representa el número total de individuos que contaban con el segmento anatómico para el registro del indicador

${ }^{1}$ Porcentajes respecto al total de individuos del mismo sexo que presentan la parte ósea para su observación

${ }^{2}$ Porcentajes respecto al total de individuos por categoría de indicador

Fuente: Datos propios

Tabla 5. Desgaste dental SJM.

\begin{tabular}{|l|c|c|c|c|c|c|c|c|c|c|}
\hline \multirow{2}{*}{ Indicador } & \multicolumn{2}{|c|}{ Mujeres } & \multicolumn{2}{|c|}{ Hombres } & \multicolumn{2}{|c|}{ Subadulto } & \multicolumn{2}{|c|}{ Adulto } & \multicolumn{2}{|c|}{ Total } \\
\cline { 2 - 11 } & $\mathbf{N}$ & $\%^{*}$ & $\mathbf{N}$ & $\%^{*}$ & $\mathbf{N}$ & $\%^{*}$ & $\mathbf{N}$ & $\%^{*}$ & $\mathbf{N}^{* *}$ & $\%^{* *}$ \\
\hline Alimentación & $10 / 11$ & 90.9 & $8 / 10$ & 90.0 & $2 / 2$ & 100 & $3 / 3$ & 100 & $23 / 26$ & 88.4 \\
\hline Actividad & $1 / 11$ & 9.0 & $2 / 10$ & 20.0 & $0 / 2$ & 0 & $0 / 3$ & 0 & $3 / 26$ & 11.5 \\
\hline
\end{tabular}

* n representa el número total de individuos del mismo sexo que contaban con el segmento anatómico para el registro del indicador

** $\mathrm{N}$ representa el número total de individuos que contaban con el segmento anatómico para el registro del indicador

${ }^{1}$ Porcentajes respecto al total de individuos del mismo sexo que presentan la parte ósea para su observación

${ }^{2}$ Porcentajes respecto al total de individuos por categoría de indicador

Fuente: Datos propios

San José Mogote (SJM) era una villa de primer orden socio-político que recibía algunos productos para su abastecimiento de otras aldeas pequeñas localizadas en sus cercanías, entre ellas Fábrica San José (FSJ), un asentamiento de tercer orden ubicado apenas a unos $5 \mathrm{~km}$ de distancia. Drennan (1976) sugiere con base en la evidencia bioarqueológica que FSJ podría haber proveído a SJM además de mano de obra, de productos como sal, madera, pieles 
y carne; en un estudio previo de Alfaro y Bernal (2009) sobre los entierros de FSJ se encontró que la mayoría de los individuos de entre 39-50 años (de ambos sexos) presentaban desgastes dentales parecidos a los aquí registrados, los cuales se relacionaban con el procesamiento de pieles, evidenciando la activa participación de las personas de edad mayor de esa aldea en una de las fases de esta tarea. En el caso de SJM, la baja presencia de individuos con desgastes de este tipo, nos lleva a pensar que si bien dentro de la aldea existían algunas personas de edad avanzada que pudieron haber colaborado en el ablandamiento de pieles, la mayoría de las pieles que se usaban en el caserío eran adquiridas de las aldeas cercanas de menor rango.

\section{CONCLUSIONES}

Los resultados aún son considerados como preliminares, debido a que aún se sigue trabajando con los materiales de fechados para el clásico y posclásico y se continúa esperando la publicación de resultados del contexto arqueológico de todos los entierros del sitio arqueológico que permita profundizar diversos aspectos de la sociedad mogoteña. Sin embargo, con base en el análisis aquí expuesto, podemos decir que si bien existen algunas particularidades sociales que indudablemente incidieron de manera diferencial en distintos aspectos de la organización social, la división del trabajo o las condiciones de salud de los antiguos pobladores de esta villa, las diferencias biológicas entre los individuos relacionadas con el sexo y la edad, jugaron también un papel importante en las variaciones de la susceptibilidad encontradas.

Si bien la división del trabajo en la aldea es factible que pudiera basarse principalmente en género, edad y habilidades personales, por lo que es posible que existieron algunas actividades que eran predominantemente desarrolladas por cierto grupo de edad o sexo, es probable también que existiera una cierta flexibilidad en la organización del trabajo, en donde los miembros de las unidades domésticas colaborarían de manera coordinada con base en su edad y habilidades en algunas fases del proceso productivo, sobre todo en ciertas épocas del año. En comunidades agrícolas de Oaxaca, puede aún observarse que la clasificación de las actividades suele estar frecuentemente en función de la energía física que requieren, de manera que la mayoría de los trabajos considerados como "pesados" son realizados por hombres mientras que los considerados "ligeros" 29 normalmente son llevados a cabo por mujeres, niños y ancianos, independientemente que sean agrícolas o desarrollados en la unidad doméstica, aunque llegan a observarse algunas variaciones en los instrumentos con los que realizan las tareas o la esfera de distribución de los productos finales (Ramos, 1998).

En el caso de las mujeres de San José Mogote, éstas es probable que hayan gozado de los mismos privilegios y privaciones de los hombres adscritos a su clase social, ya que no se encontraron claras distinciones en cuanto al tipo de enterramiento y objetos ofrendados entre hombres y mujeres que reflejaran una notoria diferencia de prestigio que pudiera estar relacionada con el sexo de los individuos, sin embargo, si se observo una tendencia a dar más importancia a destacar la pertenencia a un linaje de élite familiar (destacando al familiar que perteneciera al linaje más noble) más que al descendiente de la línea masculina de uno de los linajes divinos de Tierra y Cielo. No obstante, en el caso del poder político, la participación de las mujeres no es del todo clara, no hay evidencia suficiente para especificar su rol en este sentido, aunque Marcus y Flannery (2001) refieren con base en la evidencia arqueológica que pareciera haber una distinción en cuanto a los espacios utilizados para rituales que haría pensar en que las mujeres tenían poca injerencia en el ámbito público o de gobierno.

29. Aunque no por considerarse ligeros significa que no requieran de esfuerzo o fortaleza para llevarse acabo, podría quizás variar la intensidad y tiempo en el que se desarrollan pero esto podría llegar a compensarse con la acumulación de otras actividades desarrolladas por los individuos, cuya suma vuelve de igual manera pesados estos trabajos considerados ligeros. 
Por otro lado, si como Marcus y Flannery afirman: "uno de los métodos usados por los líderes de San José Mogote para la construcción de alianzas fue la hipogamia que consistía en enviar a una mujer de alcurnia de un centro señorial a casarse con el dirigente de una comunidad subordinada" (2001:136), entonces las mogoteñas de igual manera parecen haber jugado un papel importante en las alianzas matrimoniales que los nobles efectuaban para fortalecer su poderío o conseguir mantener el dominio sobre vastas extensiones de tierras y de recursos durante este período de rápido crecimiento de la aldea y el establecimiento de importantes redes de comercio tanto locales como con centros regionales distantes. Lo anterior se sustenta con algunos entierros femeninos de la fase Guadalupe (850-700 a.C.), que fueron los más ricos, tanto en ofrenda mortuoria como en el tipo de casa y productos artesanales de que disponían (Marcus y Flannery, 2001).

Por último, concordamos con el planteamiento de Rodríguez-Shadow (2007b) quien refiere que las mujeres mesoamericanas de la etapa aldeana realizaban las labores que la sociedad les asignaba de acuerdo con el contexto geográfico, su estatus y grupo étnico, colaborando activamente en las actividades de subsistencia y algunas actividades especializadas de tiempo parcial, pero también continuaron encargándose de las tareas del hogar, el cuidado, la educación de los infantes y la trasmisión de costumbres e ideología.

\section{REFERENCIAS BIBLIOGRÁFICAS}

Acuña, R. (1984). Relaciones Geográficas del Siglo XVI: Antequera. Tomos 1 y 2. México: Universidad Nacional Autónoma de México.

Alfaro, M.E. (2006). Tradición Funeraria Las

Palmas. Aproximación a la heterogeneidad en cazadores-recolectores-pescadores de la región costera del Golfo de Baja California Sur, México (1200-1700 d.C.). Tesis de Maestría en Antropología Física no publicada. Escuela Nacional de Antropología e Historia. México.
Alfaro, M. E., \& Bernal, F. N. (2009). Fábrica San José. Una aldea del formativo Oaxaqueño. En Estudios de Antropología Biológica XIV: 185-203, México: CONACULTA-INAH-UNAM-AMAB. Armelagos, G. (1998). Introduction: sex, gender and death status in prehistoric and contemporary populations. En Grauer, A.L. y StuartMacadam, P. (Eds.). Sex and gender in paleopathological perspective. (pp. 1-10). United Kingdom: Cambridge University Press.

Aufderheide, A. C., \& Rodríguez, M. C. (1998). The Cambridge encyclopedia of human paleopathology. United Kingdom: Cambridge University Press.

Blanton, R. E., Feinman, G., Kowalewski, S.A. \& Nicholas, L. M. (1999). Ancient Oaxaca: Cambridge University Press. USA.

Blom, D. E., Buikstra, J. E., Keng, L., Toczack, P.D., \& Shoreman, E. (2005). Anemia and childhood mortality: latitudinal patterning along the coast of pre-colombian Peru. American Journal of Physical Anthropology. 127: 152-169.

Dahlgren, B. (1990). La mixteca: su cultura e historia prehispánica. México: Universidad Nacional Autónoma de México.

De la Vega D, Sánchez Pérez, S., Hernández del Olmo, J., Ánimas, R.E., Hernández Zarza, N., Zaavedra, C. E. \& Segura, A, R. (2005). Las mujeres alfareras de los Reyes Metzontla. México: CONACULTA-INAH.

Del Paso \& Troncoso, F. (1905). Papeles de la Nueva España. Geografía y estadística (Segunda Serie). Relaciones Geográficas de la diócesis de Oaxaca. Manuscritos de la Real Academia de la Historia de Madrid y del Archivo de Indias de Sevilla. Años 1579-1581. Tomo 1 y 2. Madrid: Tipográfico Sucesores de Rivadeneyra.

Díaz-Andreu M., Lucy, S., Babic, S. \& Edwards, D.N. (2005). The archaeology of identity. Approaches to gender, age, estatus, ethnicity and religión. London: Routledge.

Drennan, R.D. (1976 ). Fabrica San José and middle Formative society in the Valley of Oaxaca. En Flannery, K.V. y Joyce, J. (Eds.) Memoirs of the Museum of Anthropology University of Michigan 8. Ann Arbor. 
Fernández Dávila, E. \& Gómez Serafín, S. (1997). Arqueología y arte. Evolución de los zapotecos de los Valles Centrales. Periodo formative. En Historia del arte de Oaxaca. Arte prehispánico 1. (pp. 79-106). Oaxaca: Instituto Oaxaqueño de las Culturas, Gobierno del Estado de Oaxaca.

Flannery, K.V, \& Marcus, J. (1983). The growth of site hierarchies in the Valley of Oaxaca: Part I. En Flannery, K.V. y Marcus, J. (eds.) The Cloud People. (pp. 53-64). New York: Academia Press.

Flannery, K.V. \& Marcus, J. (2005). Excavations at San José Mogote 1. The household archaeology. En Marcus, J. y Flannery, K.V. (Eds). Prehistory and ecology of the Valley of Oaxaca. Memoirs of the Museum of Anthropology University of Michigan 40. Ann Arbor.

Franco, P. (1580). Relación de Miaguatlan, que es de encomendero, Acuña, R. (1984). Relaciones Geográficas del Siglo XVI: Antequera. Tomo 1. México: Universidad Nacional Autónoma de México.

Gónzales Licón, E. \& Zamora Sánchez, A. (2007). Sexo y género en el valle de Oaxaca. Expresión antropológica 29: 54-80.

Goodman, A. \& Martin, D. (2002). Recostructing profiles from skeletal remains. En Steckel, R.H. y Rose. J.C. The Backbone of history. Health and Nutrition in the Western Hemisphere. (pp. 11-60) United Kingdom: Cambridge University Press.

Hershkovitz, I., Bedfor, L., Jellema, L. M. \& Latimer, B. (1996). Injuries to the Skeleton due to prolonged Activity in Hand-to-Hand Combat. International Journal of Osteoarchaeology. 6: 167-178.

Hillson S. (2002). Dental Anthropology. United Kingdom: Cambridge University Press.

Lai, P. y Lovell, N.C. (1992). Skeletal Markers of Occupational Stress in the Fur Trade: a Case Study from Hudson's Bay Company Fur Trade Post. International Journal of Ostearchaeology. 2: 221-234.

Lovell, N.C. (1997). Trauma Analysis in Paleopathology. Yearkbook of Physical Anthropology. 40: 139-170.
Lukacs, J. \& Pastor, R.F. (1988). AcitivyInduced Patterns of Dental Abrasion in Prehistoric Pakistan: Evidence from

Mehgarh and Harappa. American Journal of Physical Anthropology. 76: 377-398.

Marcus, J. (1998). Women's ritual in formative Oaxaca. Figurine-making, divination, death and the ancestors. En Marcus, J. y Flannery, K.V. (Eds.). Prehistory and ecology of the Valley of Oaxaca. Memoirs of the Museum of Anthropology University of Michigan 33. Ann Arbor.

Marcus, J. \& Flannery, K.V. (1994). Ancient zapoteco ritual and religion an application of the direct historical approach. En Renfrew, C. y Zubrow, B.W. (Eds.) The ancient mind elements of cognitive archaeology (pp. 55-75). New York: Cambridge University Press.

Marcus, J. y Flannery, K.V. (2001). La Civilización Zapoteca. Cómo evolucionó la Sociedad Urbana en el Valle de Oaxaca. México: Fondo de Cultura Económica.

Merbs, C.F. (1983). Patterns of Activityinduced Pathology in Canadian Inuit population. Archaeological Survey of Canada. 119. National Museum of Man, Mercurie Paper.

Miralles Marrarero R.C. (2001). Valoración del daño corporal del aparato locomotor. España: Editorial Elsevier.

Miralles Marrarero R.C. y Miralles Rull, I. (2007). Biomecánica clínica de las patologías del aparato locomotor. España: Editorial Elsevier.

Marrodán Serrano, M.D., González Montero de Espinosa, M., y Prado Martínez, C. (1995). Antropología de la nutrición. Técnicas, métodos y aplicaciones. España: Editorial Noesis.

Nelson, S.M. (2004). Gender in archaeology. Analysing power and prestige. Walnut Creek, CA: AltaMira Press.

Ortega Muñoz, A. (1998). La estimación de la edad en los restos óseos subadultos mesoamericanos. Colección osteológica de San Gregorio Atlapulco, Xochimilco. Tesis de Licenciatura en Antropología Física no publicada. Escuela Nacional de Antropología e Historia. México. 
Ortner, D.J. y Putchar, W.G.J. (1981). Identification of pathological conditions in human skeletal remains. Washington: Smithsonian Institution Press. Pires-Ferreira, J.W. (1976). Shell and iron-ore mirror exchange in formative Mesoamerica, with comments on others comodities. En Flannery, K.V. (ed.). The early Mesoamerican villages. (pp. 311-328). New York: Academic Press.

Ramos P.D. (1998). San Francisco Cajonos. Una comunidad campesino-indígena de la sierra norte de Oaxaca. En Ramos. P.D. (Coord). Salud $y$ tradiciones reproductivas en la sierra norte de Oaxaca. (pp. 31-68). México: Universidad Autónoma Benito Juárez de Oaxaca.

Rodríguez-Shadow, M. (1988). La mujer Azteca. México: Universidad Autónoma del Estado de México.

Rodríguez-Shadow, M. (2007a). La Arqueología de género en México. Avances y perspectivas. Expresión antropológica 29: 40-53. Gobierno del Estado de México. México.

Rodríguez-Shadow, M. (2007b). Las relaciones de género en el México prehispánico. En Rodríguez-Shadow, M. (coord.) Las mujeres en Mesoamérica prehispánica (pp. 49-78). México: Universidad Autónoma del Estado de México.

Smith, P. (1972). Diet and attrition in the Natufians. American Journal of Physical Anthropology 37: 233-238.

Soafer J.R. (2006). The body as material culture. A theorical osteoarchaeology. New York: Cambridge University Press.

Speth, J.D. (1990). Seasonality, resource stress and food sharing in so-called "egalitarian" foraging societies. Journal of anthropological archaeology 9: 148-188.

Spiemann, C.A. (1989). A review: dietary restrictions on hunter-gatherer women and the implications for fertility and infant mortality. Human Ecology 17: 321-345.

Standen, V.G. y Arriaza, B.T. (2000). Trauma in the preceramic coastal populations of northern Chile: Violence or occupational hazards? American Journal of Physical Anthropology 112: 239-249.

Steckel, R.H. y Rose, J.C. (Eds.) (2002). The backbone of history. Health and nutrition in the western hemisphere. United Kingdom: Cambridge University Press.

Stuart-Macadam, P. (1998). Iron deficiency anemia: exploring the difference. En Grauer A. y Stuart-Macadam P. (Eds.) Sex and gender in paleopathological perspectiva. (pp. 45-63). United Kingdom: Cambridge University Press. Ubelaker, D.H. (1989). Human skeleton remains, analysis, interpretation. Manual of archaeology. Taraxacum. Washington.

White D. T. y Pieter A. F. (2005). The Human Bone Manual. Amsterdam: Elsevier Acadeic Press.

Whiting, W.C. \& Zernicke, R. F. (1998). Biomechanics of Musculoskeletal Injury. Human Kinetics. First Editon. Champaign. USA.

Wiesheu, W. (2006). Arqueología de género y patrón de especialización artesanal. Cuicuilco13, 139-149.

Walker, P.L. (1986). Porotic hiperostosis in a marine-dependent California Indian population. American Journal of Physical Anthropology 69, 345-354.

Winter, M. (1990). Oaxaca Prehispánica: Una introducción. En Winter, M. (Comp.). Lecturas Históricas del estado de Oaxaca 1. Época Prehispánica (pp. 31-219). Colección Regiones de México. México: Instituto Nacional de Antropología e Historia, Gobierno de Oaxaca. 